\title{
Role of Maternal Thyroid-Stimulating Immunoglobulin in Graves' Disease for Predicting Perinatal Thyroid Dysfunction
}

\author{
Yiwen Cui, MD ${ }^{1}$ Asha Rijhsinghani, MD ${ }^{1,2}$ \\ ${ }^{1}$ Department of Obstetrics and Gynecology, Albany Medical Center, \\ Albany, New York \\ 2 Division of Maternal-Fetal Medicine, Department of Obstetrics and \\ Gynecology, Fetal Diagnosis Unit, University of lowa, lowa City, lowa \\ Address for correspondence Yiwen Cui, MD, Department of \\ Obstetrics and Gynecology, Albany Medical Center, 391 Myrtle \\ Avenue, Albany, NY 12208 (e-mail: cuiy@amc.edu).
}

Am J Perinatol Rep 2019;9:e341-e345.

\begin{abstract}
Keywords

- TSI

- Graves' disease

- thyroid dysfunction
\end{abstract}

Objective To assess maternal thyroid-stimulating immunoglobulin (TSI) as a predictor of neonatal thyroid hyperthyroidism in pregnancies complicated by Graves' disease. Methods This is a 10-year retrospective study of patients with a history of Graves' disease and elevated TSI activity level defined as 1.3 times the normal. All subjects underwent cordocentesis for ultrasound findings of suspected fetal thyrotoxicosis (fetal tachycardia, oligohydramnios, hydrops, and thyromegaly). Neonatal diagnosis was made based on neonatal thyroid function testing or symptoms.

Results Fourteen patients were included in the study, seven with active Graves' disease requiring antithyroid drug ("ATD group") and seven with iatrogenic hypothyroidism on levothyroxine ("levothyroxine group"). Four cases (57\%) of neonatal thyrotoxicosis were diagnosed in the levothyroxine group compared with two cases (28\%) in the ATD group. The lowest maternal TSI level at which a neonate did not develop hyperthyroidism was 2.6 for the levothyroxine group and 2.5 for the ATD group. The odds ratio of a neonate from the levothyroxine group developing hyperthyroidism compared with one from the ATD group is 3.3 (95\% confidence interval: 0.4-30.7).

Conclusion For patients with Graves' disease, those with iatrogenic hypothyroidism and TSI $>2.5$ times the basal level are at the highest risk for neonatal thyrotoxicosis.
Thyroid disorders are the most common endocrinopathies in women of reproductive age, with overall prevalence for hyperthyroidism in the United States at $1.2 \%{ }^{1}$ During pregnancy, hyperthyroidism is less common at 0.1 to $0.4 \%$, but Graves' disease accounts for $85 \%$ of these cases. ${ }^{2,3}$

Graves' disease poses significant risks to the pregnancy and the fetus. Patients with hyperthyroidism in pregnancy are at increased risk of spontaneous abortion, fetal growth restriction, and stillbirth. ${ }^{4,5}$ Neonates born to these mothers exhibit hyperthyroidism in 2 to $5 \%$ of cases, which can present immediate and long-term challenges including cardiac insufficiency, liver dysfunction, and psychomotor disabilities. ${ }^{6-9}$

Assessment of fetal thyroid dysfunction is difficult in utero, as it can involve invasive procedures such as cordocentesis in addition to serial ultrasounds. Studies have investigated maternal thyroid autoimmune antibodies as an alternative method in predicting fetal thyroid function. The antibodies have been shown to be persistently elevated despite successful treatment of Graves' disease ${ }^{10}$ and can cross the placenta to influence fetal thyroid activity. ${ }^{11}$ received

February 17, 2019 accepted after revision May 6, 2019
DOI https://doi.org/

10.1055/s-0039-1694035. ISSN 2157-6998.
Copyright $\odot 2019$ by Thieme Medical Publishers, Inc., 333 Seventh Avenue, New York, NY 10001, USA. Tel: +1(212) 584-4662.
License terms

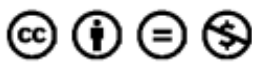


Much attention has focused on the role of thyroid-stimulating hormone (TSH) receptor antibodies (TRAb). The American Thyroid Association recommends close monitoring of fetal status for patients who have a maternal TRAb level three times the upper limit of normal. ${ }^{12}$ However, TRAb refers to the general class of immunoglobulin binding to the TSH receptor, which includes thyroid-stimulating immunoglobulin (TSI) and blocking antibodies. ${ }^{13}$ Graves' disease is directly associated with the function of TSI, and thus, this marker may be a more sensitive predicator for neonatal hyperthyroidism.

There is currently no specific reference level defining the elevation of maternal TSI at which the fetus is at increased risk for thyroid dysfunction. The aim of this study is to determine the threshold maternal TSI level above which additional fetal monitoring is needed to detect perinatal hyperthyroidism. We also assessed the value of cordocentesis in patients with elevated TSI.

\section{Methods}

We conducted a retrospective study over a 10 -year period at the Fetal Diagnosis and Treatment Center at the University of Iowa that focused on patients who had a history of Graves' disease and an elevated TSI level at the initial establishment of prenatal care. Those who underwent cordocentesis for reasons of abnormal ultrasound findings were included in the study. All mothers were managed and kept at the euthyroid state.

In cases clinically suspected of fetal thyroid dysfunction, the women were counseled regarding the option of cordocentesis for confirmation of the fetal diagnosis. Indications included sustained fetal tachycardia, intrauterine growth restriction (IUGR) (estimated fetal weight $<10$ th percentile), oligohydramnios (amniotic fluid index $<5 \mathrm{~cm}$ ), fetal hydrops, or gross fetal thyromegaly. In utero diagnosis of thyroid dysfunction were based on reference ranges of free T4 (FT4) and TSH established by Thorpe-Beeston et al's study. ${ }^{14}$

After delivery, cord blood was collected for thyroid function tests (TSH, FT4, and TSI). Subsequent neonatal evaluation and management depended on the clinical assessment of the attending neonatologists. Neonatal hypothyroidism was defined as FT4 below the 5th percentile and TSH above the 95th percentile. Neonatal hyperthyroidism (or thyrotoxicosis) was diagnosed by FT4 above the 95th percentile and TSH below the 5th percentile. Treatment of neonatal thyroid dysfunction was at the discretion of the neonatologists based on neonatal thyroid assessment.

TSI was quantified in sera using a commercial semiquantitative bioassay (TSI Assay, ARUP Laboratories, Salt Lake City, UT). The value for TSI is represented as a multiple above the basal activity level of the immunoglobulin. All other maternal and neonatal thyroid markers (FT4, TSH) were determined using an in-house automated immunoassay analyzer.

Data was analyzed with Minitab Statistical Software Version 18 (State College, PA).

\section{Results}

We identified 14 women with a history of Graves' disease who underwent indicated cordocentesis ( - Table 1 ). Seven of the 14 participants had active disease during pregnancy requiring treatment with an antithyroid drug (ATD) (referred to as "ATD group"); the other seven who became hypothyroid following prior radioiodine therapy were on levothyroxine during pregnancy ("levothyroxine group").

All participants had an elevated TSI level above the threshold of 1.3 , which represents $133 \%$ above the basal activity level. The 1.3 is the current maternal cutoff above which a patient is diagnosed with Graves' disease. ${ }^{15}$ The average TSI for the hyperthyroid group was elevated at 12.0 and for the hypothyroid group at 16.8. Cordocentesis was performed in the second trimester when indicated by abnormal ultrasound findings. Fetal tachycardia was the most common abnormality across both groups with 7 of 14 cases. Interestingly, oligohydramnios and IUGR were only seen in the maternal levothyroxine group, while two cases of thyromegaly and one of pericardial effusion were observed in the ATD group.

The diagnosis of fetal thyroid dysfunction based on cordocentesis matched the neonatal outcome for 11 of 14 cases (79\%). Six neonates were diagnosed with hyperthyroidism (6 of 14, 43\%), 4 from the levothyroxine group and 2 from the ATD group. All six neonates required treatment with ATD.

Four neonates (cases 1, 3-5) in the maternal levothyroxine group received in utero treatment because of fetal hyperthyroidism diagnosed through cordocentesis. The mothers were prescribed propylthiouracil (PTU) in the antepartum period at the discretion of the physician. Three of the four $(75 \%)$ neonates continued to be hyperthyroid after delivery.

Six preterm deliveries ( 6 of $14,43 \%$ ) occurred from $30^{0 / 7}$ to $36^{6 / 7}$ gestational weeks. Four were from the levothyroxine group, while two were from the ATD group. All other deliveries were at term. There was no neonatal demise in the study.

The lowest maternal TSI level at which the infant did not develop hyperthyroidism was 2.6 for the levothyroxine group and 2.5 for the ATD group.

\section{Discussion}

Neonatal hyperthyroidism has mostly been described in the context of maternal Graves' disease. In theory, the disease process is initiated by the transfer of maternal TSI to the fetal circulation, leading to increased fetal thyroid activity. TRAb is the hallmark of Graves' disease and while titers may fluctuate depending of treatment, studies have shown that $\sim 10 \%$ of patients remain TRAb positive after medical or surgical treatment. ${ }^{10,16}$

Encompassed in the TRAb class of antibodies are two types of immunoglobulins, TSI and thyroid blocking antibodies. TSI directly activates the TSH receptor and leads to increase in thyroid hormone secretion. ${ }^{17,18}$ Thus, transplacental passage of these antibodies can lead to in utero 


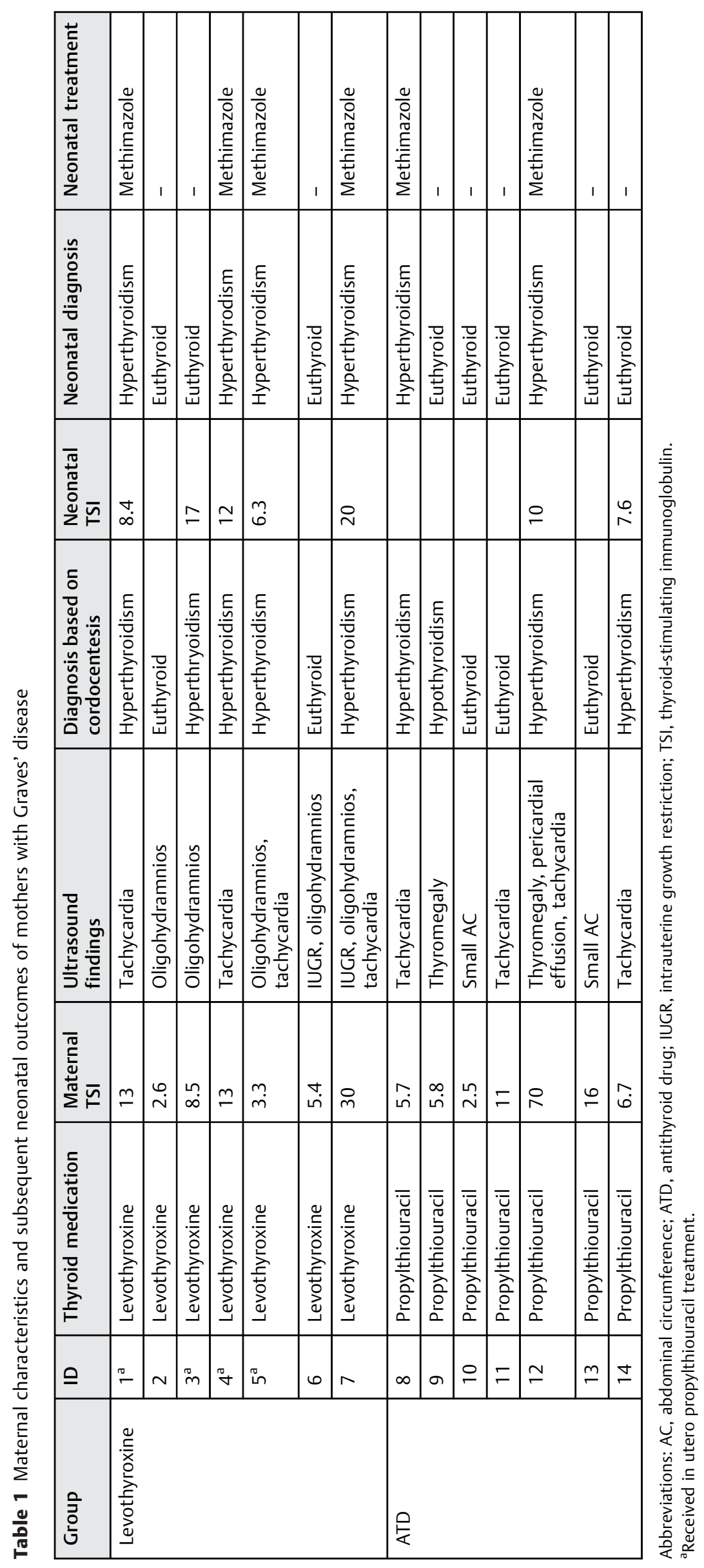


thyrotoxicosis that continue into the neonatal period until the disappearance of the transmitted antibodies, usually within the first 3 months of life. ${ }^{19}$

The bulk of literature on correlation between maternal thyroid antibodies and the development of neonatal thyrotoxicosis have focused on TRAb. ${ }^{20-22}$ There are few published reports on TSI and its effect on the perinatal outcome. ${ }^{11,19,23}$ To our knowledge, our study of 14 patients who underwent cordocentesis is the largest study that systematically assessed the relationship of maternal TSI and its impact on neonatal thyroid dysfunction.

We found that the threshold of 2.6 was the maternal TSI level below which neonatal thyroid dysfunction was not diagnosed in the levothyroxine group and 2.5 for the ATD group. The two thresholds are comparable, suggesting that a universal TSI threshold level can be set at 2.5 regardless of maternal history. The value 2.5 represents the TSI activity level that is $250 \%$ above the basal TSI activity.

However, our study also demonstrates that maternal thyroid history should not be overlooked. More cases of neonatal thyrotoxicosis were observed in the maternal levothyroxine group than the ATD group. The odds ratio of a neonate diagnosed with hyperthyroidism from a mother on levothyroxine compared with a neonate from a mother on ATD was 3.3 (95\% confidence interval: 0.4-30.7). This may be partially explained by the transfer of ATD across the placenta. ${ }^{24,25}$ The fetuses in the ATD group of our study would have incidentally been receiving treatment in utero. Maternal transfer of ATD can also delay the diagnosis of neonatal thyrotoxicosis as the drug takes up to 7 to 10 days to be eliminated from the neonatal system. ${ }^{26}$ However, in our study population, the diagnose of euthyroidism remained unchanged in the neonatal period. Our findings suggest that maternal treatment history for Graves' disease should be taken into account when monitoring patients with elevated TSI, particularly if the patient received radioiodine treatment in the past.

Four fetuses in the study were intentionally treated in utero, all in the maternal levothyroxine group. The mothers were prescribed PTU based on diagnosis of fetal hyperthyroidism through cordocentesis and the doses were titrated based on repeated cordocentesis values. However, three of the four treated fetuses persisted to have thyrotoxicosis in the neonatal period requiring treatment. The mixed response in our study to in utero fetal hyperthyroid treatment is consistent with the lack of evidence-based strategy to dose PTU. In previously published cases of in utero treatment, variable neonatal outcomes were observed with a majority of infants still exhibiting symptoms of thyrotoxicosis. ${ }^{27,28}$

The limitations of this study include the possible lack of generality from being conducted at a single tertiary institution. Management of maternal and fetal status was further individualized at the discretion of the physician. However, our findings are based on objective data acquired through detailed longitudinal follow-up. Despite the retrospective nature, our study correlates neonatal outcome with maternal data and in utero fetal testing, which can be difficult to replicate in a prospective manner.

\section{Conclusion}

While cordocentesis appears to be accurate in prenatal diagnosis of fetal hyperthyroidism, it does not help with the management of the disease. As there lacks an adequate in utero treatment for fetal thyroid dysfunction, maternal TSI and maternal history may enable physicians to identify highrisk populations at preconception to optimize their care and outcome. We found that mothers with Graves' disease and a TSI level of 2.5 or above have an increased risk of giving birth to neonates with thyrotoxicosis. This risk can be further stratified by the type of maternal treatment for Graves' disease, with the highest clinical suspicion for mothers with iatrogenic hypothyroidism.

\section{Conflict of Interest}

The authors have no conflict of interest to declare.

\section{Acknowledgment}

The authors would like to acknowledge University of Iowa and Albany Medical Center for supporting the conduction of this research.

\section{References}

1 Girling JC. Thyroid disorders in pregnancy. Obstetrics, Gynaecol Reprod Med 2008;18(10):259-264

2 Patil-Sisodia K, Mestman JH. Graves hyperthyroidism and pregnancy: a clinical update. Endocr Pract 2010;16(01):118-129

3 Burch HB, Cooper DS. Management of Graves disease: a review. JAMA 2015;314(23):2544-2554

4 Kriplani A, Buckshee K, Bhargava VL, Takkar D, Ammini AC. Maternal and perinatal outcome in thyrotoxicosis complicating pregnancy. Eur J Obstet Gynecol Reprod Biol 1994;54(03):159-163

5 Millar LK, Wing DA, Leung AS, Koonings PP, Montoro MN, Mestman JH. Low birth weight and preeclampsia in pregnancies complicated by hyperthyroidism. Obstet Gynecol 1994;84(06): 946-949

6 Mitsuda N, Tamaki H, Amino N, Hosono T, Miyai K, Tanizawa O. Risk factors for developmental disorders in infants born to women with Graves disease. Obstet Gynecol 1992;80(3 Pt 1): 359-364

7 Polak M. Hyperthyroidism in early infancy: pathogenesis, clinical features and diagnosis with a focus on neonatal hyperthyroidism. Thyroid 1998;8(12):1171-1177

8 Polak M, Le Gac I, Vuillard E, et al. Fetal and neonatal thyroid function in relation to maternal Graves' disease. Best Pract Res Clin Endocrinol Metab 2004;18(02):289-302

9 Daneman D, Howard NJ. Neonatal thyrotoxicosis: intellectual impairment and craniosynostosis in later years. J Pediatr 1980; 97(02):257-259

10 McKenzie JM, Zakarija M. Fetal and neonatal hyperthyroidism and hypothyroidism due to maternal TSH receptor antibodies. Thyroid 1992;2(02):155-159

11 Hamada N, Momotani N, Ishikawa N, et al. Persistent high TRAb values during pregnancy predict increased risk of neonatal hyperthyroidism following radioiodine therapy for refractory hyperthyroidism. Endocr J 2011;58(01):55-58

12 Alexander EK, Pearce EN, Brent GA, et al. 2017 Guidelines of the American Thyroid Association for the diagnosis and management of thyroid disease during pregnancy and the postpartum. Thyroid 2017;27(03):315-389

13 Latif R, Morshed SA, Zaidi M, Davies TF. The thyroid-stimulating hormone receptor: impact of thyroid-stimulating hormone and 
thyroid-stimulating hormone receptor antibodies on multimerization, cleavage, and signaling. Endocrinol Metab Clin North Am 2009;38(02):319-341, viii

14 Thorpe-Beeston JG, Nicolaides KH, Felton CV, Butler J, McGregor AM. Maturation of the secretion of thyroid hormone and thyroidstimulating hormone in the fetus. N Engl J Med 1991;324(08): 532-536

15 Thyroid stimulating immunoglobulin. ARUP Laboratories. Available at: http://Itd.aruplab.com/test/pub/0099430. Accessed March 24, 2018

16 Laurberg P, Wallin G, Tallstedt L, Abraham-Nordling M, Lundell G, Tørring O. TSH-receptor autoimmunity in Graves' disease after therapy with anti-thyroid drugs, surgery, or radioiodine: a 5-year prospective randomized study. Eur J Endocrinol 2008;158(01): 69-75

17 Zakarija M, McKenzie JM. Pregnancy-associated changes in the thyroid-stimulating antibody of Graves' disease and the relationship to neonatal hyperthyroidism. J Clin Endocrinol Metab 1983; 57(05):1036-1040

18 Levy-Shraga Y, Tamir-Hostovsky L, Boyko V, Lerner-Geva L, Pinhas-Hamiel O. Follow-up of newborns of mothers with Graves' disease. Thyroid 2014;24(06):1032-1039

19 van Dijk MM, Smits IH, Fliers E, Bisschop PH. Maternal thyrotropin receptor antibody concentration and the risk of fetal and neonatal thyrotoxicosis: a systematic review. Thyroid 2018;28(02):257-264

20 Clavel S, Madec AM, Bornet H, Deviller P, Stefanutti A, Orgiazzi J. Anti TSH-receptor antibodies in pregnant patients with autoim- mune thyroid disorder. Br J Obstet Gynaecol 1990;97(11): 1003-1008

21 Barbesino G, Tomer Y. Clinical review: clinical utility of TSH receptor antibodies. J Clin Endocrinol Metab 2013;98(06): 2247-2255

22 Bjørgaas MR, Farstad H, Christiansen SC, Blaas HG. Impact of thyrotropin receptor antibody levels on fetal development in two successive pregnancies in a woman with Graves' disease. Horm Res Paediatr 2013;79(01):39-43

23 Momotani N, Noh JY, Ishikawa N, Ito K. Effects of propylthiouracil and methimazole on fetal thyroid status in mothers with Graves' hyperthyroidism. J Clin Endocrinol Metab 1997;82(11): 3633-3636

24 Duncombe GJ, Dickinson JE. Fetal thyrotoxicosis after maternal thyroidectomy. Aust N Z J Obstet Gynaecol 2001;41(02):224-227

25 Zakarija M, McKenzie JM, Hoffman WH. Prediction and therapy of intrauterine and late-onset neonatal hyperthyroidism. J Clin Endocrinol Metab 1986;62(02):368-371

26 Volumenie JL, Polak M, Guibourdenche J, et al. Management of fetal thyroid goitres: a report of 11 cases in a single perinatal unit. Prenat Diagn 2000;20(10):799-806

27 Aslam M, Inayat M. Fetal and neonatal Graves disease: a case report and review of the literature. South Med J 2008;101(08): 840-841

28 Nachum Z, Rakover Y, Weiner E, Shalev E. Graves' disease in pregnancy: prospective evaluation of a selective invasive treatment protocol. Am J Obstet Gynecol 2003;189(01):159-165 